\title{
Copeland Voting Fully Resists Constructive Control*
}

\author{
Piotr Faliszewski \\ Department of Computer Science \\ University of Rochester \\ Rochester, NY 14627 \\ Lane A. Hemaspaandra \\ Department of Computer Science \\ University of Rochester \\ Rochester, NY 14627
}

\author{
Edith Hemaspaandra ${ }^{\dagger}$ \\ Department of Computer Science \\ Rochester Institute of Technology \\ Rochester, NY 14623 \\ Jörg Rothe \\ Institut für Informatik \\ Heinrich-Heine-Universität Düsseldorf \\ 40225 Düsseldorf, Germany
}

Univ. of Rochester Comp. Sci. Dept. Technical Report TR-2007-923

October 21, 2007

\begin{abstract}
Control and bribery are settings in which an external agent seeks to influence the outcome of an election. Faliszewski et al. [7] proved that Llull voting (which is here denoted by Copeland ${ }^{1}$ ) and a variant (here denoted by Copeland ${ }^{0}$ ) of Copeland voting are computationally resistant to many, yet not all, types of constructive control and that they also provide broad resistance to bribery. We study a parameterized version of Copeland voting, denoted by Copeland ${ }^{\alpha}$, where the parameter $\alpha$ is a rational number between 0 and 1 that specifies how ties are valued in the pairwise comparisons of candidates in Copeland elections. We establish resistance or vulnerability results, in every previously studied control scenario, for Copeland ${ }^{\alpha}$ for each rational $\alpha, 0<\alpha<1$. In particular, we prove that Copeland ${ }^{0.5}$, the system commonly referred to as "Copeland voting," provides full resistance to constructive control. Among the systems with a polynomial-time winner problem, this is the first natural election system proven to have full resistance to constructive control. Results on bribery and fixed-parameter tractability of bounded-case control proven for Copeland ${ }^{0}$ and Copeland ${ }^{1}$ in $[7]$ are extended to Copeland ${ }^{\alpha}$ for each rational $\alpha, 0<\alpha<1$; we also give results in more flexible models such as microbribery and extended control.
\end{abstract}

\section{Introduction}

Preference aggregation by voting procedures has been the focus of much attention within the field of multiagent systems. Agents (called voters in the context of voting) may have different, often conflicting individual preferences over the given alternatives (or candidates). Voting rules (or, synonymously, election systems) provide a useful method for them to come to a "reasonable" decision

\footnotetext{
*Supported in part by DFG grant RO-1202/9-3 and RO-1202/11-1, NSF grants CCR-0311021, CCF-0426761, and IIS-0713061, the Alexander von Humboldt Foundation's TransCoop program, and a Friedrich Wilhelm Bessel Research Award.

${ }^{\dagger}$ Work done in part while visiting Heinrich-Heine-Universität Düsseldorf.

${ }^{\ddagger}$ Work done in part while visiting the University of Rochester.
} 
on which alternative to choose. One key issue here is that there might be attempts to influence the outcome of elections. Settings in which such influence on elections can be implemented include manipulation [3, 13], electoral control [2, 7, 8, 9, 13], and bribery [6, 7]. Although reasonable election systems typically are susceptible to these kinds of influence (for manipulation this is universally true, via the Gibbard-Satterthwaite and Duggan-Schwartz Theorems), computational complexity can be used to provide some protection in each such setting. We study the extent to which the Copeland election system [4] (see also [14, 11]) resists, computationally, control and bribery attempts.

Copeland elections are one of the classical voting procedures that are based on pairwise comparisons of candidates: The winner (by a strict majority of votes) of each such a head-to-head contest is awarded one point and the loser receives no point; whoever collects the most points over all these contests (including tie-related points) is the election's winner. The points awarded for ties in such head-to-head majority-rule contests are treated in various ways in the literature. Faliszewski et al. [7] proposed a parameterized version of Copeland elections, denoted by Copeland ${ }^{\alpha}$, where the parameter $\alpha$ is a rational number between 0 and 1 such that, in case of a tie, both candidates receive $\alpha$ points. So the system widely referred to in the literature as "Copeland elections" is Copeland ${ }^{0.5}$, where tied candidates receive half a point each (see, e.g., Merlin and Saari [14, 11]; the definition used by Conitzer et al. [3] can be scaled to be equivalent to Copeland ${ }^{0.5}$ ). Copeland ${ }^{0}$, where tied candidates come away empty-handed, has sometimes also been referred to as "Copeland elections" (see, e.g., $[12,7]$ ). An election system proposed by the Catalan philosopher and theologian Ramon Llull in the 13th century (see, e.g., the references in [7]) is in this notation nothing other than Copeland $^{1}$, where tied candidates are awarded one point each, just like winners of head-to-head contests.

Faliszewski et al. [7] studied the systems Copeland ${ }^{0}$ and Copeland $^{1}$ with respect to their (computational) resistance and/or vulnerability to bribery and procedural control. Bribery and control are settings in which an external actor seeks to influence the outcome of an election. Bribery is somewhat akin to electoral manipulation and strategic voting in that the briber tries to reach his or her goal by bribing some voters to change their preferences. (The difference between bribery and manipulation is that manipulative voters themselves cast their votes insincerely, i.e., there is no external agent.) In contrast, the external actor in control scenarios (who by tradition is, potentially confusingly, called "the chair") seeks to reach this goal via changing the election procedure, namely via adding/deleting/partitioning either candidates or voters.

Bartholdi, Tovey, and Trick [2] were the first to study the computational aspects of control: How hard is it, computationally, for the chair to exert control? In their seminal paper they introduced a number of fundamental control scenarios involving (what is now called) constructive control, i.e., where the chair's goal is to make some designated candidate win. Other papers studying control include $[8,13,9,7]$, which in addition to constructive control also consider destructive control, where the chair tries to preclude some designated candidate from winning. The notion of bribery in elections was introduced by Faliszewski et al. [6] and was also studied in [7].

At first glance, one might be tempted to think that the definitional perturbation due to the parameter $\alpha$ in Copeland ${ }^{\alpha}$ elections is negligible. However, as noted in [7], “... it can make the dynamics of Llull's system quite different from those of [Copeland ${ }^{0}$. Proofs of results for Llull differ considerably from those for [Copeland ${ }^{0}$." This statement notwithstanding, we show that in most cases it is possible to obtain a unified - though sometimes rather involved - construction that works for both systems, and even for Copeland ${ }^{\alpha}$ with respect to every rational $\alpha, 0 \leq \alpha \leq 1$. 
In particular, we establish resistance or vulnerability results for Copeland ${ }^{0.5}$ (which is the system commonly referred to as "Copeland") in every previously studied control scenario. In doing so, we provide an example of a control problem where the complexity of Copeland ${ }^{0.5}$ differs from that of both Copeland ${ }^{0}$ and Copeland ${ }^{1}$ : While the latter two problems are vulnerable to constructive control by adding (an unlimited number of) candidates, Copeland ${ }^{0.5}$ is resistant to this control type (see Section 2 for definitions and Theorem 3.7 for this result).

Thus Copeland (i.e., Copeland ${ }^{0.5}$ ) is the first natural election system with a polynomial-time winner problem that is proven to be resistant to every type of constructive control that has been proposed in the literature to date. Moreover, if one uses the hybridization method of Hemaspaandra et al. [9] to combine this full resistance of Copeland ${ }^{0.5}$ to constructive control with the full resistance of Copeland $d^{0.5}$ to destructive voter control (which we also prove here) and with the full resistance of plurality ${ }^{1}$ to destructive candidate control (see $[8,7]$ ), one obtains a hybrid election system that (a) is resistant to every (constructive and destructive) control type previously considered in the literature, (b) has a polynomial-time winner problem, and (c) has only natural election systems as its constituents. In contrast, one of the constituent systems for the hybrid constructed in [9], which is there shown to resist twenty control types, is rather artificial.

\section{Preliminaries}

An election is specified by a finite set $C$ of candidates and a finite collection $V$ of voters, where each voter has preferences over the candidates. We consider both rational and irrational voters. The preferences of a rational voter are expressed by a preference list of the form $a>b>c$ (assuming $C=\{a, b, c\})$, where the underlying relation $>$ is a strict linear order that is transitive. The preferences of an irrational voter are expressed by a preference table that, for any two distinct candidates, specifies which of them is preferred to the other by this voter. An election system is a rule that determines the winner(s) of each given election $(C, V)$. In this paper, we consider a parameterized version of Copeland's election system [4], denoted Copeland ${ }^{\alpha}$, where the parameter $\alpha$ is a rational number between 0 and 1 that specifies how ties are rewarded in the head-to-head majority-rule contests between any two distinct candidates.

Definition 2.1 ([7]) Let $\alpha, 0 \leq \alpha \leq 1$, be a fixed rational number. In a Copeland ${ }^{\alpha}$ election, the voters indicate which among any two distinct candidates they prefer. For each such head-to-head contest, if some candidate is preferred by a strict majority of voters then he or she obtains one point and the other candidate obtains zero points, and if a tie occurs then both candidates obtain $\alpha$ points. Let $E=(C, V)$ be an election. For each $c \in C$, score ${ }_{E}^{\alpha}(c)$ is the sum of $c^{\prime} s$ Copeland $^{\alpha}$ points in E. Every candidate $c$ with maximum score ${ }_{E}^{\alpha}(c)$ wins.

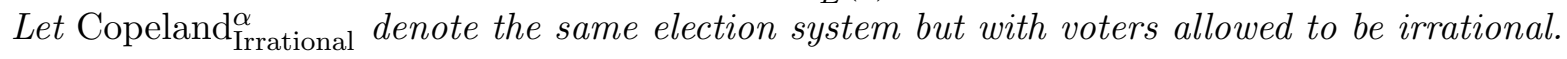

In the literature, the term "Copeland elections" is most often used for the system Copeland ${ }^{0.5}$, and is sometimes used for Copeland ${ }^{0}$. The system Copeland ${ }^{1}$ was proposed by Llull already in the 13 th century (see the references in [7]) and so is called Llull voting.

We now define the control problems we consider, in both the constructive and the destructive version. Let $\mathcal{E}$ be an election system. In our case, $\mathcal{E}$ will be either Copeland ${ }^{\alpha}$ or Copeland Irrational $^{\alpha}$,

\footnotetext{
${ }^{1}$ In plurality-rule elections, every voter gives one point to his or her most preferred candidate. Whoever collects the most points is this election's plurality winner.
} 
where $\alpha, 0 \leq \alpha \leq 1$, is a fixed rational number. In fact, since the types of control we consider here are well-known from the literature (see, e.g., $[2,7,8]$ ), we will content ourselves with the definition of some examples of these problems (in particular some of those that occur in the proofs to be presented in Section 3.2 below).

We start with defining control via adding candidates. Note that there are two versions of this control type. The unlimited version (which, for the constructive case, was introduced by Bartholdi, Tovey, and Trick [2]) asks whether the election chair can add (any number of) candidates from a given pool of spoiler candidates in order to either make his or her favorite candidate win the election (in the constructive case), or prevent his or her despised candidate from winning (in the destructive case):

Name: $\mathcal{E}-\mathrm{CCAC}_{\mathrm{u}}$ and $\mathcal{E}-\mathrm{DCAC}_{\mathrm{u}}$.

Given: Disjoint candidate sets $C$ and $D$, a collection $V$ of voters represented via their preference lists (or preference tables in the irrational case) over the candidates in $C \cup D$, and a distinguished candidate $p \in C$.

Question $\left(\mathcal{E}-\mathrm{CCAC}_{\mathrm{u}}\right)$ : Does there exist a subset $D^{\prime}$ of $D$ such that $p$ is a winner of the $\mathcal{E}$ election with candidates $C \cup D^{\prime}$ and voters $V$ ?

Question $\left(\mathcal{E}-\mathrm{DCAC}_{\mathrm{u}}\right)$ : Does there exist a subset $D^{\prime}$ of $D$ such that $p$ is not a winner of the $\mathcal{E}$ election with candidates $C \cup D^{\prime}$ and voters $V$ ?

The only difference in the limited version of constructive and destructive control via adding candidates $(\mathcal{E}$-CCAC and $\mathcal{E}$-DCAC, for short) is that the chair needs to achieve his or her goal by adding at most $k$ candidates from the given set of spoiler candidates. This version of control by adding candidates was proposed in [7] to synchronize the definition of control by adding candidates with the definitions of control by deleting candidates, adding voters, and deleting voters.

Our second example regards control via run-off partition of candidates, where we focus on the constructive case:

Name: $\mathcal{E}$-CCRPC-TP (respectively, $\mathcal{E}$-CCRPC-TE).

Given: A set $C$ of candidates and a collection $V$ of voters represented via their preference lists (or preference tables in the irrational case) over $C$, a distinguished candidate $p \in C$, and a nonnegative integer $k$.

Question: Is it possible to partition $C$ into $C_{1}$ and $C_{2}$ such that $p$ is a winner of the two-stage election where the winners of subelection $\left(C_{1}, V\right)$ that survive the tie-handling rule (TP or $\mathrm{TE})$ compete against the winners of subelection $\left(C_{2}, V\right)$ that survive the tie-handling rule? (Subelections are conducted using system $\mathcal{E}$.)

As one can see from the above examples, we use the following naming conventions for control problems. The name of a control problem starts with the election system used (when clear from context, it may be dropped), followed by CC for "constructive control" or by DC for "destructive control," followed by the acronym of the type of control: AC for "adding (a limited number of) candidates," $\mathrm{AC}_{\mathrm{u}}$ for "adding (an unlimited number of) candidates," DC for "deleting candidates," PC for "partition of candidates," RPC for "run-off partition of candidates," AV for "adding voters," 
DV for "deleting voters," and PV for "partition of voters," and all the partitioning cases (PC, RPC, and PV) are followed by the acronym of the tie-handling rule used in subelections, namely TP for "ties promote" (i.e., all winners of a given subelection are promoted to the final round of the election) and TE for "ties eliminate" (i.e., if there is more than one winner in a given subelection then none of this subelection's winners is promoted to the final round of the election).

We now turn to the definition of bribery problems (see [6]), where the briber seeks to reach his or her goal via bribing certain voters to make them change their preferences.

Name: $\mathcal{E}$-bribery.

Given: A set $C$ of candidates, a collection $V$ of voters represented via their preference lists (or preference tables in the irrational case) over $C$, a distinguished candidate $p \in C$, and a nonnegative integer $k$.

Question: Does there exist a voter collection $V^{\prime}$ over $C$, where $V^{\prime}$ results from $V$ by modifying at most $k$ voters, such that $p$ wins the $\mathcal{E}$ election $\left(C, V^{\prime}\right)$ ?

For $\mathcal{E}$-destructive-bribery, the destructive bribery problem for $\mathcal{E}$, we require $p$ to be not a winner.

Note that the above definitions focus on a winner, i.e., they are in the nonunique-winner model. The unique-winner analogs of these problems can be defined by requiring the distinguished candidate $p$ to be the unique winner (or to not be a unique winner in the destructive case).

Let $\mathcal{E}$ be an election system and let $\Phi$ be a control type. We say $\mathcal{E}$ is immune to $\Phi$-control if the chair can never reach his or her goal (of making a given candidate win in the constructive case, and of blocking a given candidate from winning in the destructive case) via asserting $\Phi$-control. $\mathcal{E}$ is said to be susceptible to $\Phi$-control if $\mathcal{E}$ is not immune to $\Phi$-control. $\mathcal{E}$ is said to be vulnerable to $\Phi$-control if it is susceptible to $\Phi$-control and there is a polynomial-time algorithm for solving the control problem associated with $\Phi$. $\mathcal{E}$ is said to be resistant to $\Phi$-control if it is susceptible to $\Phi$-control and the control problem associated with $\Phi$ is NP-hard. The above notions were introduced by Bartholdi, Tovey, and Trick [2] (see also, e.g., $[8,13,9,7]$ ). We say $\mathcal{E}$ is vulnerable to constructive (respectively, destructive) bribery if $\mathcal{E}$-bribery (respectively, $\mathcal{E}$-destructive-bribery) is in $\mathrm{P}$. We say $\mathcal{E}$ is resistant to constructive (respectively, destructive) bribery if $\mathcal{E}$-bribery (respectively, $\mathcal{E}$-destructive-bribery) is NP-hard.

Many of our reductions in Section 3 are from the NP-complete vertex cover problem: Given an undirected graph $G=(V(G), E(G))$ and a nonnegative integer $k$, does there exist a set $W$ such that $W \subseteq V(G),\|W\| \leq k$, and for every edge $e=\{u, v\}, e \in E(G)$, it holds that $e \cap W \neq \emptyset$ ?

The study of fixed-parameter complexity (see, e.g., [5]) has been expanding explosively since it was parented as a field by Downey, Fellows, and others in the late 1980s and the 1990s. Although the area has built a rich variety of complexity classes regarding parameterized problems, for the purpose of the current paper we need focus only on one very important class, namely, the class FPT. Briefly put, a problem parameterized by some value $j$ (which, note, can be viewed as a family of problems, one per value of $j$ ) is said to be fixed-parameter tractable (equivalently, to belong to the class FPT) if for each fixed value of $j$ there is an $\mathcal{O}\left(n^{c}\right)$ algorithm for the problem, where $c$ is a constant independent of $j$. (The constant implicit in the "big-oh," however, may depend on $j$.)

In our context, we consider two parameterizations: bounding the number of candidates and bounding the number of voters. We use the same notations used throughout this paper to describe problems, except we postpend a "- $\mathrm{BV}_{j}$ " to a problem name to state that the number of voters may 


\begin{tabular}{|l|c|c|c|c|c|c|}
\hline \multirow{2}{*}{} & \multicolumn{5}{|c|}{ Copeland $^{\alpha}$} \\
\cline { 2 - 7 } & \multicolumn{2}{|c|}{$\alpha=0$} & \multicolumn{2}{|c|}{$0<\alpha<1$} & \multicolumn{2}{|c|}{$\alpha=1$} \\
\hline Control type & $\mathrm{CC}$ & $\mathrm{DC}$ & $\mathrm{CC}$ & $\mathrm{DC}$ & $\mathrm{CC}$ & $\mathrm{DC}$ \\
\hline $\mathrm{AC}_{\mathrm{u}}$ & $\mathrm{V}$ & $\mathrm{V}$ & $\mathbf{R}$ & $\mathbf{V}$ & $\mathrm{V}$ & $\mathrm{V}$ \\
$\mathrm{AC}$ & $\mathrm{R}$ & $\mathrm{V}$ & $\mathbf{R}$ & $\mathbf{V}$ & $\mathrm{R}$ & $\mathrm{V}$ \\
DC & $\mathrm{R}$ & $\mathrm{V}$ & $\mathbf{R}$ & $\mathbf{V}$ & $\mathrm{R}$ & $\mathrm{V}$ \\
RPC-TP & $\mathrm{R}$ & $\mathrm{V}$ & $\mathbf{R}$ & $\mathbf{V}$ & $\mathrm{R}$ & $\mathrm{V}$ \\
RPC-TE & $\mathrm{R}$ & $\mathrm{V}$ & $\mathbf{R}$ & $\mathbf{V}$ & $\mathrm{R}$ & $\mathrm{V}$ \\
PC-TP & $\mathrm{R}$ & $\mathrm{V}$ & $\mathbf{R}$ & $\mathbf{V}$ & $\mathrm{R}$ & $\mathrm{V}$ \\
PC-TE & $\mathrm{R}$ & $\mathrm{V}$ & $\mathbf{R}$ & $\mathbf{V}$ & $\mathrm{R}$ & $\mathrm{V}$ \\
\hline PV-TE & $\mathrm{R}$ & $\mathrm{R}$ & $\mathbf{R}$ & $\mathbf{R}$ & $\mathrm{R}$ & $\mathrm{R}$ \\
PV-TP & $\mathrm{R}$ & $\mathrm{R}$ & $\mathbf{R}$ & $\mathbf{R}$ & $\mathrm{R}$ & $\mathrm{R}$ \\
AV & $\mathrm{R}$ & $\mathrm{R}$ & $\mathbf{R}$ & $\mathbf{R}$ & $\mathrm{R}$ & $\mathrm{R}$ \\
DV & $\mathrm{R}$ & $\mathrm{R}$ & $\mathbf{R}$ & $\mathbf{R}$ & $\mathrm{R}$ & $\mathrm{R}$ \\
\hline
\end{tabular}

Table 1: Resistance (R) and vulnerability (V) of Copeland ${ }^{\boldsymbol{\alpha}}$ elections, for rationals $\boldsymbol{\alpha}, \mathbf{0} \leq \boldsymbol{\alpha} \leq \mathbf{1}$.

be at most $j$, and we postpend a "- $\mathrm{BC}_{j}$ " to a problem name to state that the number of candidates may be at most $j$. In each case, the bound applies to the full number of such items involved in the problem. For example, in the case of control by adding voters, the $j$ must bound the total of the number of voters in the election added together with the number of voters in the pool of voters available for adding.

\section{Control}

\subsection{Overview of Results}

Our main result regarding control is Theorem 3.1 below.

Theorem 3.1 For each rational $\alpha, 0 \leq \alpha \leq 1$, Copeland ${ }^{\alpha}$ elections are resistant and vulnerable to control as shown in Table 1, both for rational and irrational voters and in both the nonunique-winner model and the unique-winner model.

Boldface results in Table 1 are new to this paper and nonboldface results are due to Faliszewski et al. [7]. Note that the notion widely referred to in the literature simply as "Copeland elections," which we here for clarity call Copeland ${ }^{0.5}$, possesses all ten of our basic types of constructive resistance and, in addition, even has constructive $\mathrm{AC}_{\mathrm{u}}$ resistance. These resistances should be compared with the results known for the other notion that in the literature is occasionally referred to as "Copeland elections," namely Copeland ${ }^{0}$, and with the results known for Llull elections, which are here denoted by Copeland ${ }^{1}$, see [7]. While Copeland ${ }^{0}$ and Copeland ${ }^{1}$ possess all ten of our basic types of constructive resistance, they both are vulnerable to this eleventh type of constructive control, the incongruous but historically resonant notion of constructive control by adding an unlimited number of candidates (i.e., $\mathrm{CCAC}_{\mathrm{u}}$ ).

It is known that plurality is resistant to the six basic types of destructive candidate control and also to $\mathrm{DCAC}_{\mathrm{u}}$, see $[8,7]$. Since by Theorem 3.1, Copeland ${ }^{0.5}$ provides resistance for all ten basic constructive control types and for $\mathrm{CCAC}_{\mathrm{u}}$, and also for the four basic types of destructive voter 
control, the hybrid (in the sense of [9]) of plurality with Copeland ${ }^{0.5}$ is resistant to each basic type of constructive and destructive control and in addition to constructive and destructive $\mathrm{AC}_{\mathrm{u}}$ control. This result follows via Theorem 3.1 and the results of Hemaspaandra et al. [9]. And, unlike the hybrid system constructed by Hemaspaandra et al. [9], this hybrid uses only natural systems as its constituents.

Corollary 3.2 The hybrid (in the sense of [9]) of plurality and Copeland ${ }^{0.5}$ is resistant to each of the twenty basic types of constructive and destructive control and also to constructive and destructive $A C_{\mathrm{u}}$ control, and it has a polynomial-time winner problem.

The next two sections discuss the single results contained in Theorem 3.1 in more detail and sketch some of the proofs. All the results stated in Sections 3.2 and 3.3 are true both in the rational and irrational voter model and in both the nonunique-winner model and the unique-winner model.

\subsection{Candidate Control}

We start with candidate control. Faliszewski et al. [7] showed that both Copeland ${ }^{0}$ and Copeland ${ }^{1}$ are vulnerable to each destructive control type in Table 1. To extend these results to Copeland ${ }^{\alpha}$ elections for each rational $\alpha, 0 \leq \alpha \leq 1$, our proofs for destructive control by adding and deleting candidates use the following observation. Let $(C, V)$ be an election, and let $\alpha$ be a fixed rational number such that $0 \leq \alpha \leq 1$. For every candidate $c \in C$ it holds that: $\operatorname{score}_{(C, V)}^{\alpha}(c)=\sum_{d \in C-\{c\}} \operatorname{score}_{(\{c, d\}, V)}^{\alpha}(c)$.

The candidate partition and run-off partition cases can be shown to reduce to the case of deleting candidates, and the vulnerability results in Theorem 3.3 use greedy algorithms.

Theorem 3.3 For each rational number $\alpha, 0 \leq \alpha \leq 1$, Copeland ${ }^{\alpha}$ is vulnerable to destructive control via (a) adding candidates (both $D C A C$ and $D C A C_{\mathrm{u}}$, i.e., both for a limited and an unlimited number of candidates), (b) deleting candidates $(D C D C),(c)$ partition of candidates (in both the TP and TE model, i.e., DCPC-TP and DCPC-TE), and (d) run-off partition of candidates (in both the TP and TE model, i.e., DCRPC-TP and DCRPC-TE),

Turning now to constructive candidate control, our resistance proofs use the following two lemmas, which we here state without proof. Lemma 3.4 shows how to construct a "padded" election with useful properties. Lemma 3.5 then shows how to build an election via combining smaller ones.

Lemma 3.4 Let $\alpha$ be a rational number such that $0 \leq \alpha \leq 1$. For each positive integer $n$, there is an election $\operatorname{Pad}_{n}=(C, V)$ such that $\|C\|=2 n+1$ and, for each candidate $c \in C$, it holds that score $_{\mathrm{Pad}_{n}}^{\alpha}(c)=n$.

Lemma 3.5 Let $E=(C, V)$ be an election where $C=\left\{c_{1}, \ldots, c_{n}\right\}$, and let $\alpha$ be a rational number such that $0 \leq \alpha \leq 1$. For each candidate $c_{i}$, we denote the number of head-to-head ties of $c_{i}$ in $E$ by $t_{i}$. Let $k_{1}, \ldots, k_{n}$ be a sequence of n nonnegative integers such that for each $k_{i}$ we have $0 \leq k_{i} \leq n$. There is an election $E^{\prime}=\left(C^{\prime}, V^{\prime}\right)$ such that: (a) $C^{\prime}=C \cup D$, where $D=\left\{d_{1}, \ldots, d_{2 n^{2}}\right\}$; (b) for each $i, 1 \leq i \leq n$, score $E^{\prime}\left(c_{i}\right)=2 n^{2}-k_{i}+\alpha t_{i}$; (c) for each $i, 1 \leq i \leq 2 n^{2}$, $\operatorname{score}_{E^{\prime}}^{\alpha}\left(d_{i}\right) \leq n^{2}+1$. 
Faliszewski et al. [7] showed that both Copeland ${ }^{0}$ and Copeland $^{1}$ are resistant to constructive control via adding (a limited number of) candidates. This is subsumed by the following more general result.

Theorem 3.6 For each rational number $\alpha$ such that $0 \leq \alpha \leq 1$, Copeland ${ }^{\alpha}$ is resistant to constructive control via adding candidates (CCAC).

In contrast with the known result that both Copeland ${ }^{0}$ and Copeland ${ }^{1}$ are vulnerable to constructive control via adding an unlimited number of candidates [7], we show that Copeland ${ }^{\alpha}$ is resistant to this control type if $0<\alpha<1$.

Notation: In the proofs of Section 3.2, we often identify an election with its set of candidates, since in the case of candidate control the set of voters is fixed and cannot change.

Theorem 3.7 For each rational number $\alpha, 0<\alpha<1$, Copeland ${ }^{\alpha}$ is resistant to constructive control via adding an unlimited number of candidates $\left(C C A C_{\mathrm{u}}\right)$.

Proof. We provide a reduction from the vertex cover problem. Let $(G, k)$ be an instance of the vertex cover problem, where $G$ is an undirected graph and $k$ is the bound on the size of the vertex cover that we seek. Let $E(G)=\left\{e_{1}, \ldots, e_{m}\right\}$ be the set of $G$ 's edges and $V(G)=\{1, \ldots, n\}$ be the set of $G$ 's vertices. Using Lemma 3.5, we can build an election $E^{\prime}=\left(C, V^{\prime}\right)$ such that: (a) $\|C\|=2 \ell^{2}+\ell$, where $\ell=2 n+2 m$; (b) $\left\{p, r, e_{1}, \ldots, e_{m}\right\} \subseteq C$ (the remaining candidates are used for padding); (c) $\operatorname{score}_{E^{\prime}}^{\alpha}(p)=2 \ell^{2}-2$; (d) $\operatorname{score}_{E^{\prime}}^{\alpha}(r)=2 \ell^{2}-2-k+k \alpha$ in the nonunique-winner case (respectively, score $E_{E^{\prime}}^{\alpha}(r)=2 \ell^{2}-2-k+(k-1) \alpha$ in the unique-winner case); (e) for each $e_{i} \in C$, $\operatorname{score}_{E^{\prime}}^{\alpha}\left(e_{i}\right)=2 \ell^{2}-2+\alpha$ in the nonunique-winner case (respectively, $\operatorname{score}_{E^{\prime}}^{\alpha}\left(e_{i}\right)=2 \ell^{2}-2$ in the unique-winner case); (f) the scores of all candidates other than $p, e_{1}, \ldots, e_{m}$ are at most $2 \ell^{2}-n-2$.

(We omit the details of the construction, but mention that one can start from any election with at least $2 n+2 m$ candidates, add some ties between some padding candidates and the $e_{i}$ 's if needed, and then apply Lemma 3.5.)

We form election $E=(C \cup D, V)$ from $E^{\prime}$ via adding candidates $D=\{1, \ldots, n\}$ and appropriate voters such that the results of head-to-head contests are: (1) $p$ ties with all candidates in $D ;(2)$ for each $e_{j}$, if $e_{j}$ is incident with $i \in D$ then candidate $i$ defeats candidate $e_{j}$, and otherwise they tie; (3) all other candidates in $C^{\prime}$ defeat each of the candidates in $D$.

Our instance of $\mathrm{CCAC}_{\mathrm{u}}$ is formed by the candidate set $C$ (the candidates already enrolled in the election), the candidate set $D$ (the candidates that can be added to the election), and the set of voters $V$, where each voter has preferences over the candidates in $C \cup D$. Note that the candidates in $D$ correspond to the vertices of $G$. We claim that there is a set $D^{\prime}$ (where $D^{\prime} \subseteq D$ ) such that $p$ is a winner (respectively, the unique winner) of the Copeland ${ }^{\alpha}$ election $\left(C \cup D^{\prime}, V\right)$ if and only if $G$ has a vertex cover of size at most $k$.

It is easy to see that if $D^{\prime}$ corresponds to a vertex cover of size at most $k$ then $p$ is a winner (respectively, the unique winner) of Copeland ${ }^{\alpha}$ election $C \cup D^{\prime}$. The reason is that adding any one member of $D^{\prime}$ increases $p$ 's score by $\alpha$, increases $r$ 's score by one, and for each $e_{j}$, adding $i \in D^{\prime}$ increases $e_{j}$ 's score by $\alpha$ if and only if $e_{j}$ is not incident with $i$. Thus, the nonpadding candidates in $C \cup D^{\prime}$ have the following scores in the resulting election $E^{\prime \prime}$ with candidates $C \cup D^{\prime}$ (it is clear that none of the padding candidates has enough Copeland ${ }^{\alpha}$ points to become a winner after adding any subset of candidates from $D$ ): (a) $\operatorname{score}_{E^{\prime \prime}}^{\alpha}(p)=2 \ell^{2}-2+k \alpha$; (b) $\operatorname{score}_{E^{\prime \prime}}^{\alpha}(r)=2 \ell^{2}-2+k \alpha$ in the nonunique-winner case (respectively, $2 \ell^{2}-2+(k-1) \alpha$ in the unique-winner case); (c) 
$\operatorname{score}_{E^{\prime \prime}}^{\alpha}\left(e_{i}\right) \leq 2 \ell^{2}-2+k \alpha$ in the nonunique-winner case (respectively, $2 \ell^{2}-2+(k-1) \alpha$ in the unique-winner case).

As a result, we see that adding all members of $D^{\prime}$ makes $p$ a winner (respectively, the unique winner).

On the other hand, assume that $p$ can become a winner via adding some subset $D^{\prime}$ of candidates from $D$. First, note that $\left\|D^{\prime}\right\| \leq k$, since otherwise $r$ would end up with more points (respectively, at least as many points) as $p$ and so $p$ would not be a winner (respectively, would not be the unique winner). We claim that $D^{\prime}$ corresponds to a vertex cover of $G$. For the sake of contradiction assume that there is some edge $e_{j}$ incident to vertices $u$ and $v$ such that neither $u$ nor $v$ is in $D^{\prime}$. However, if this were the case then candidate $e_{j}$ would have more points (respectively, at least as many points) as $p$ and so $p$ would not be a winner (respectively, would not be the unique winner). Thus, $D^{\prime}$ must form a vertex cover of size at most $k$.

The following result extends to all rationals $\alpha, 0 \leq \alpha \leq 1$, the known result that Copeland ${ }^{0}$ and Copeland ${ }^{1}$ are resistant to constructive control via deleting candidates [7].

Theorem 3.8 Let $\alpha$ be a rational number such that $0 \leq \alpha \leq 1$. Copeland ${ }^{\alpha}$ is resistant to constructive control via deleting candidates $(C C D C)$.

Proof. The proof follows via a reduction from the vertex cover problem. We first handle the nonunique-winner case.

Let $(G, k)$ be the input instance of the vertex cover problem, where $G$ is an undirected graph and $k$ is the upper bound on the size of the vertex cover that we seek. Let $V(G)=\{1, \ldots, n\}$ and let $E(G)=\left\{e_{1}, \ldots, e_{m}\right\}$. We build election $E^{\prime}=\left(C^{\prime}, V^{\prime}\right)$, where $C^{\prime}=\left\{p, r, e_{1}, \ldots, e_{m}, 1, \ldots, n\right\}$ and the voter set $V^{\prime}$ yields the following results of head-to-head contests (omitting the details of the construction due to space): (1) $p$ defeats $r$; (2) each candidate $e_{i} \in C$ defeats exactly those two candidates $u, v \in\{1, \ldots, n\}$ that the edge $e_{i}$ is incident with; (3) each candidate $u \in\{1, \ldots, n\}$ defeats both $p$ and all candidates $e_{i}$ such that vertex $u$ is not incident to $e_{i}$; (4) all the other contests result in a tie.

Let $\ell=n+m$. We form an election $E=(C, V)$ via combining election $E^{\prime}$ with election $\operatorname{Pad}_{\ell}=\left(C^{\prime \prime}, V^{\prime \prime}\right)$, where $C^{\prime \prime}=\left\{t_{0}, \ldots, t_{2 \ell}\right\}$ and the set $V^{\prime \prime}$ of voters is set as in Lemma 3.4. We select the following results of head-to-head contest between the candidates in $C^{\prime}$ and the candidates in $C^{\prime \prime}$ : $p$ and all candidates $e_{i} \in C$ defeat everyone in $C^{\prime \prime}$ and each candidate in $C^{\prime \prime}$ defeats all candidates

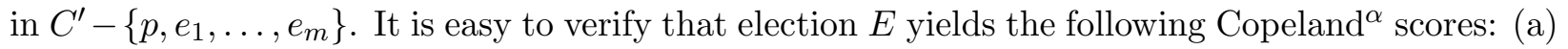
$\operatorname{score}_{E}^{\alpha}(p)=m \alpha+1+2 \ell+1$; (b) score ${ }_{E}^{\alpha}(r)=m+n \alpha$; (c) for each $e_{i} \in C, \operatorname{score}_{E}^{\alpha}\left(e_{i}\right)=m \alpha+2+2 \ell+1$; (d) for each $i \in C$, $\operatorname{score}_{E}^{\alpha}(i) \leq 1+m+n \alpha$; (e) for each $t_{i} \in C, \operatorname{score}_{E}^{\alpha}\left(t_{i}\right)=\ell+n+1$.

Thus, the set of winners of $E$ is $W=\left\{e_{1}, \ldots, e_{m}\right\}$. We claim that $p$ can become a winner of Copeland $^{\alpha}$ election $E$ via deleting at most $k$ candidates if and only if the graph $G$ has a vertex cover of size at most $k$.

First note that if $k \geq n$ then $G$ obviously has a vertex cover of size at most $k$ (namely, the set of all the vertices) and so from now on we assume $k<n$. Also, it is easy to see that if $k \geq m$ (i.e., if our vertex cover can have more elements than there are edges) then clearly a vertex cover exists and so we assume that $k<m$. Also, we note that all candidates except $p$ lose by at least $n+1$ points to each of the winners and so $p$ is the only candidate that can possibly become a winner via deleting at most $k \leq n$ candidates.

Assume that $p$ can become a winner via deleting at most $k<n$ candidates, and let $D \subseteq C$ be a smallest set such that deleting exactly the candidates in $D$ from election $E$ guarantees $p$ 's 
victory. We start by observing that $D$ necessarily contains only candidates that correspond to vertices of $G$. For the sake of contradiction, assume that $D$ does contain some candidate $d$ such that $d \notin\{1, \ldots, n\}$. Clearly, $d$ cannot be $r$, since deleting $r$ decreases $p$ 's score without changing the score of any of the candidates in $W$ and so removing $r$ from $D$ would yield a smaller set of candidates whose deletion guarantees $p$ 's victory. Similarly, $d$ cannot be any other non-vertex candidate, since deleting $d$ from election $E$ would affect the score of $p$ and the scores of all remaining candidates from $\left\{e_{1}, \ldots e_{m}\right\}$ in the same way. ${ }^{2}$ Thus, again, removing $d$ from $D$ would yield a smaller set with the required property.

Now note that, in election $E$, each of $e_{1}, \ldots, e_{m}$ has exactly one Copeland ${ }^{\alpha}$ point of advantage over $p$. Deleting any candidate $u$ corresponding to a vertex of $G$ does not affect $p$ 's score but it does lower by one the score of all the candidates $e_{1}, \ldots, e_{m}$ that correspond to the edges incident with $u$. Since deleting the candidates in $D$ makes $p$ a winner and since $D$ contains only up to $k$ candidates that correspond to vertices in $G$, it must be the case that the candidates in $D$ correspond to a vertex cover of $G$ having size at most $k$.

For the converse, it is easy to see that if $G$ has a vertex cover of size at most $k$ then deleting the candidates that correspond to this vertex cover guarantees $p$ 's victory. This completes the proof for the nonunique-winner case.

To obtain the proof for the unique-winner case, we need to add one more candidate, $\hat{r}$, that is a "clone" of $r$ (i.e., $\hat{r}$ ties in the head-to-head contest with $r$ and has the same results as $r$ in all other head-to-head contests). In such a modified election, $p$ has the same Copeland ${ }^{\alpha}$ score as each of the $e_{i}$ 's and has to gain at least one point over each of them to become the unique winner. The rest of the argument remains the same.

Theorem 3.8 will be helpful in treating the (run-off) partition-of-candidates cases. Again, it is known from [7] that both Copeland ${ }^{0}$ and Copeland ${ }^{1}$ are resistant to constructive control by (run-off) partition of candidates in both the ties-promote model and the ties-eliminate model.

Theorem 3.9 Let $\alpha$ be a rational number such that $0 \leq \alpha \leq 1$. Copeland ${ }^{\alpha}$ is resistant to constructive control via run-off partition of candidates in both the ties-promote model (CCRPC-TP) and the ties-eliminate model (CCRPC-TE).

Proof. Our proof will, again, follow via a reduction from the vertex cover problem. Our input is a graph $G$ and a nonnegative integer $k$ and we seek an election $E$ where our favorite candidate $p$ can be made a winner in the CCRPC-TP (respectively, CCRPC-TE) model if and only if $G$ contains a vertex cover of size at most $k$. Let $G$ have the edge set $E(G)=\left\{e_{1}, \ldots, e_{m}\right\}$ and the vertex set $V(G)=\{1, \ldots, n\}$. The following construction is the basis of our proof.

Construction 3.10 Let $F$ and $H$ be two elections, with candidate sets $\left\{f_{1}, \ldots, f_{n}\right\}$ and $\left\{h_{1}, \ldots, h_{q}\right\}$, respectively. Define $E=(C, V)$, where $C=\left\{r, f_{1}, \ldots, f_{n}, h_{1}, \ldots, h_{q}\right\}$ and voters in $V$ are set so that we have the following results of the head-to-head contests: (1) for each $f_{i} \in C$, $f_{i}$ defeats $r$; (2) for each $h_{i} \in C, r$ defeats $h_{i}$; (3) for each $h_{i}, f_{j} \in C, h_{i}$ defeats $f_{j}$; (4) all the remaining head-to-head contests are as in $F$ and $H$, respectively.

Intuitively, Construction 3.10 works as follows. We set $F$ to be an election that contains the candidate $p$ and where $p$ can be made a winner (respectively, the unique winner) via deleting a set

\footnotetext{
${ }^{2}$ Keep in mind that $d$ could be one of the candidates $e_{1}$ through $e_{m}$. However, since $k<m, D$ cannot contain all of these candidates.
} 
$D$ of at most $k$ candidates (specifically - and importantly - we will use the elections built in the proof of Theorem 3.8). We will set the election $H$ in such a way that the only run-off partitions of candidates in $E$ that could possibly result in $p$ being a winner (respectively, the unique winner) would form two subcommittees such that the first one would contain candidates in $F$, possibly without having up to $k$ of them, and the other subcommittee would contain $r$, the candidates from $H$, and the remaining candidates in $D$. This way the problem of constructive control via run-off partition of candidate reduces to the problem of finding the set $D$, which in Theorem 3.8 we have shown to be NP-complete.

Let $F$ and $H$ be two elections where $H$ contains at least two candidates, and let $E$ be the election obtained from $F$ and $H$ using Construction 3.10. We assume that our preferred candidate, $p$, belongs to $F$, and we assume that there are no ties in head-to-head contests between candidates in $H$. Later on we will precisely specify how the elections $F$ and $H$ are built, and for now we only mention that in the TE case we will have $H$ have a unique winner.

We have the following result regarding the possible structure of the subelections in the run-off partition of candidates.

Lemma 3.11 Let $\left(C_{1}, C_{2}\right)$ be a partition of candidates in $E$ such that $p$ is a winner (respectively, the unique winner), where $p$ participates in subelection $C_{1}$. It holds that $C_{1}=F-D$ and $C_{2}=$ $H \cup D \cup\{r\}$, where $D \subseteq F-\{p\}$.

Lemma 3.11 follows directly from Lemma 3.12 below the proof of which is omitted.

Lemma 3.12 Let $\left(C_{1}, C_{2}\right)$ be a partition of candidates in $E$ such that $p$ is a winner (respectively, the unique winner). The subcommittee that contains $p$ does not contain any member of $H$ nor $r$.

Using the above lemmas, we can specify the elections $F$ and $H$ and complete the proof. We will first handle the nonunique-winner cases and then we will argue how to modify the proof to apply to the unique-winner model.

For the ties-promote (respectively, ties-eliminate) case, we set $F$ to be the election built in the proof of Theorem 3.8 for the nonunique-winner model (respectively, for the unique-winner model). For the ties-promote case, we set $H$ to be an election with candidate set $\left\{r, h_{1}, \ldots, h_{q}\right\}$ such that, for some nonnegative integer $\ell$, we have the following scores: ${ }^{3}$ (a) $\operatorname{score}_{H}^{\alpha}(r)=\ell$; (b) $\operatorname{score}_{H}^{\alpha}\left(h_{1}\right)=\ell-k-1$; (c) score ${ }_{H}^{\alpha}\left(h_{2}\right)=\ell-k-1$; (d) for each $i \in\{3, \ldots, q\}$, score ${ }_{H}^{\alpha}\left(h_{i}\right) \leq \ell-k-1$. Such an election is easy to build in polynomial time using Lemma 3.5. For the ties-eliminate case, we set $H$ to have candidate set $\left\{r, h_{1}, \ldots, h_{q}\right\}$ with the following scores: (a) $\operatorname{score}_{H}^{\alpha}(r)=\ell$; (b) score $_{H}^{\alpha}\left(h_{1}\right)=\ell-k ;(\mathrm{c})$ for each $i \in\{2, \ldots, q\}, \operatorname{score}_{H}^{\alpha}\left(h_{i}\right)<\ell-k$.

Lemma 3.13 Set D in Lemma 3.11 cannot contain more than $k$ elements.

The proof of Lemma 3.13 is omitted. To complete the proof of Theorem 3.9, note that since $p$ can become a winner of his or her subcommittee if and only if $p$ can be made a winner (respectively, the unique winner) of election $F-D$, where $D \subseteq F-\{p\}$ and $\|D\| \leq k$, we see that $p$ can become a winner (respectively, the unique winner) only if $G$ has a vertex cover of size at most $k$. This follows by our choice of $F$. On the other hand, if we choose $D$ to be the set of vertices that correspond to

\footnotetext{
${ }^{3}$ Candidate $r$ in Construction 3.10 was, strictly speaking, not a member of $H$, but for the sake of building the election $E$ here it is easier to consider members of $H$ and $r$ jointly.
} 
an at-most-size- $k$ vertex cover of $G$ and partition the candidates in $C$ as in Lemma 3.11, then $p$ is a winner (respectively, the unique winner), since if we use such a partition and the set $D$ then the subcommittee $H \cup D \cup\{r\}$ either has no winner or has the unique winner $r$, and the subcommittee $F-D$ either has the unique winner $p$ (in the TE case) or has winner set $\{p\} \cup W$ (in the TP case), where $W \subseteq\left\{e_{1}, \ldots, e_{m}\right\}$. Since $p$ and all members of $W$ are tied, they all become the winners of election $E$. This completes the proof for the nonunique-winner case.

We still need to handle the unique-winner cases. However, note that in the case of the TE model the current proof already works just as well in the unique-winner model. It remains to handle the unique-winner case in the ties-promote model. However, in this case we simply need to take $F$ to be the unique-winner version of election $E$ from Theorem 3.8. Our lemmas describing the structure of the subcommittees apply in the ties-promote case, and so if $p$ is to be a winner then $r$ should be the unique winner of subcommittee $H \cup D \cup\{r\}$, where $D$ is a subset of $F-\{p\}$ with at most $k$ elements, and the global winner of the election, if any, is the unique winner of election $F-D$. Since we know that $p$ can become a unique winner of $F$ via deleting at most $k$ candidates if and only if $G$ has a vertex cover of size at most $k$, the proof is complete.

Theorem 3.9

Finally, we state without proof our result for constructive control by partition of candidates, which extends the corresponding known result for Copeland ${ }^{0}$ from [7]. Unlike in the case of run-off partition of candidates, however, our proof does not apply to the case of $\alpha=1$ for the TE model, but note that these resistances of Copeland ${ }^{1}$ were already shown in [7] for both the TP and the TE model.

Theorem 3.14 1. Let $\alpha$ be a rational number with $0 \leq \alpha \leq 1$. Copeland $^{\alpha}$ is resistant to constructive control via partition of candidates in the ties-promote model (CCPC-TP).

2. Let $\alpha$ be a rational number with $0 \leq \alpha<1$. Copeland ${ }^{\alpha}$ is resistant to constructive control via partition of candidates in the ties-eliminate model (CCPC-TE).

\subsection{Voter Control}

Our first result regarding voter control extends to all rationals $\alpha, 0 \leq \alpha \leq 1$, the corresponding result for Copeland ${ }^{0}$ and Copeland ${ }^{1}$ from [7]. The proof is omitted.

Theorem 3.15 Let $\alpha$ be a rational number such that $0 \leq \alpha \leq 1$. Copeland ${ }^{\alpha}$ is resistant to both constructive and destructive control via adding voters (CCAV and DCAV).

Next, we state without proof our result for control by deleting voters, which extends the corresponding known result for Copeland ${ }^{0}$ from [7]. Note that our proof does not apply to the case of $\alpha=1$, but we mention that these resistances of Copeland ${ }^{1}$ were already shown in [7].

Theorem 3.16 Let $\alpha$ be a rational number such that $0 \leq \alpha<1$. Copeland ${ }^{\alpha}$ is resistant to both constructive and destructive control via deleting voters (CCDV and DCDV).

Finally, we state without proof our result for control by (run-off) partition of voters, which extends the corresponding results for Copeland ${ }^{0}$ and Copeland ${ }^{1}$ from [7].

Theorem 3.17 Let $\alpha$ be a rational number such that $0 \leq \alpha \leq 1$. Copeland ${ }^{\alpha}$ is resistant to constructive and destructive control via partition of voters (in both the TP and TE model, i.e., $C C P V-T P, C C P V-T E, D C P V-T P$, and DCPV-TE), and to run-off partition of voters (in both the $T P$ and TE model, i.e., CCRPV-TP, CCRPV-TE, DCRPV-TP, and DCRPV-TE). 


\subsection{FPT Algorithm Schemes for Bounded-Case Control}

\subsubsection{Fixed-Parameter Tractability Results}

In their seminal paper on NP-hard winner-determination problems, Bartholdi, Tovey, and Trick [1] suggested considering hard election problems for the cases of a bounded number of candidates or a bounded number of voters, and they obtained efficient-algorithm results for such cases. Within the study of elections, this same approach - seeking efficient fixed-parameter algorithm families - has also been used, for example, within the study of bribery [6]. Faliszewski et al. [7] showed that the 16 resistance results for constructive and destructive voter control within Copeland ${ }^{0}$ and Copeland ${ }^{1}$ (see Table 1) are in FPT (i.e., they each are fixed-parameter tractable) if the number of candidates is bounded, and also if the number of voters is bounded. They also showed that these results hold even when the multiplicities of preference lists in a given election are represented succinctly (by a binary number).

We extend these results in Theorems 3.18 and 3.19 below. To state these results concisely, we borrow a notational approach from transformational grammar, and use square brackets as an "independent choice" notation. So, for example, the claim $\left[\begin{array}{c}\text { It } \\ \text { She } \\ \mathrm{He}\end{array}\right]\left[\begin{array}{c}\text { runs } \\ \text { walks }\end{array}\right]$ is a shorthand for six assertions: It runs; She runs; He runs; It walks; She walks; and He walks. A special case is the symbol " $\emptyset$ " which, when it appears in such a bracket, means that when unwound it should be viewed as no text at all. For example, "[ $\underset{\emptyset}{\text { Succinct }}]$ Copeland is fun" asserts both "Succinct Copeland is fun" and "Copeland is fun."

Theorem 3.18 For each rational $\alpha, 0 \leq \alpha \leq 1$, and each choice from the independent choice brackets below, the specified problem family (as $j$ varies over $\mathbb{N}$ ) is in $\mathrm{FPT}$ :

$$
\left[\begin{array}{c}
\text { succinct } \\
\emptyset
\end{array}\right]-\left[\begin{array}{c}
\text { Copeland }^{\alpha} \\
\text { Copeland }_{\text {Irrational }}^{\alpha}
\end{array}\right]-\left[\begin{array}{c}
\mathrm{C} \\
\mathrm{D}
\end{array}\right] \mathrm{C}\left[\begin{array}{c}
\mathrm{AV} \\
\mathrm{DV} \\
\mathrm{PV}-\mathrm{TE} \\
\mathrm{PV}-\mathrm{TP}
\end{array}\right]-\left[\begin{array}{c}
\mathrm{BV}_{j} \\
\mathrm{BC}_{j}
\end{array}\right] .
$$

Theorem 3.19 For each rational $\alpha, 0 \leq \alpha \leq 1$, and each choice from the independent choice brackets below, the specified problem family (as $j$ varies over $\mathbb{N}$ ) is in FPT:

$$
\left[\begin{array}{c}
\text { succinct } \\
\emptyset
\end{array}\right]-\left[\begin{array}{c}
\text { Copeland }^{\alpha} \\
\text { Copeland Irrational }_{\text {Iral }}
\end{array}\right]-\left[\begin{array}{c}
\mathrm{C} \\
\mathrm{D}
\end{array}\right] \mathrm{C}\left[\begin{array}{c}
\mathrm{AC}_{\mathrm{u}} \\
\mathrm{AC} \\
\mathrm{DC} \\
\mathrm{PC}-\mathrm{TE} \\
\mathrm{PC}-\mathrm{TP} \\
\mathrm{RPC}-\mathrm{TE} \\
\mathrm{RPC}-\mathrm{TP}
\end{array}\right]-\mathrm{BC}_{j} .
$$

The proofs of Theorems 3.18 and 3.19, which in particular employ Lenstra's [10] algorithm for bounded-variable-cardinality integer programming, are omitted here.

\subsubsection{FPT and Extended Control}

In this section, we introduce and look at extended control. By that we do not mean changing the basic control notions of adding/deleting/partitioning candidates/voters. Rather, we mean 
generalizing past merely looking at the constructive (make a distinguished candidate a winner) and the destructive (prevent a distinguished candidate from being a winner) cases. In particular, we are interested in control where the goal can be far more flexibly specified, for example (though in the partition cases we will be even more flexible than this), we will allow as our goal region any (reasonable - there are some time-related conditions) subcollection of "Copeland outcome tables" (specifications of who won/lost/tied each head-to-head contest).

Since from a Copeland outcome table, in concert with the current $\alpha$, one can read off the Copeland ${ }_{\text {Irrational }}^{\alpha}$ scores of the candidates, this allows us a tremendous range of descriptive flexibility in specifying our control goals, e.g., we can specify a linear order desired for the candi-

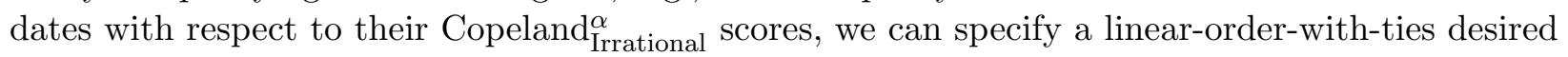

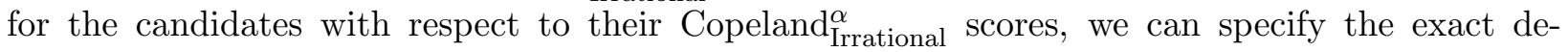
sired Copeland ${ }_{\text {Irrational }}^{\alpha}$ scores for one or more candidates, we can specify that we want to ensure that no candidate from a certain subgroup has a Copeland $\alpha_{\text {Irrational }}^{\alpha}$ score that ties or beats the Copeland $\alpha_{\text {Irrational }}^{\alpha}$ score of any candidate from a certain other subgroup, etc.

All the FPT algorithms given in the previous section regard, on their surface, the standard control problem, which tests whether a given candidate can be made a winner (constructive case) or can be precluded from being a winner (destructive case). We note that the general approaches used in that section in fact yield FPT schemes even for the far more flexible notions of control mentioned above.

\subsubsection{Resistance Results}

In contrast with the FPT results in [7] for Copeland ${ }^{0}$ and Copeland ${ }^{1}$, Faliszewski et al. [7] showed that, for $\alpha \in\{0,1\}$, Copeland $d_{\text {Irrational }}^{\alpha}$ remains resistant to all types of candidate control even for two voters. We extend these results by showing that even for each rational $\alpha, 0 \leq \alpha \leq 1$, for Copeland $_{\text {Irrational }}^{\alpha}$ all the candidate-control cases that we showed earlier in this paper (i.e., without bounds on the number of voters) to be resistant remain resistant even for the case of bounded voters. This resistance holds even when the input is not in succinct format, and so it certainly also holds when the input is in succinct format.

The only open cases remaining regard the rational-voter, candidate-control, bounded-voter cases.

\section{Bribery}

Theorem 4.1 extends to all rationals $\alpha, 0 \leq \alpha \leq 1$, the corresponding result for Copeland ${ }^{0}$ and Copeland ${ }^{1}$ from [7].

Theorem 4.1 For each rational $\alpha, 0 \leq \alpha \leq 1$, Copeland ${ }^{\alpha}$ is resistant to both constructive and destructive bribery in both the rational-voters case and the irrational-voters case.

We also extend another result for Copeland ${ }^{0}$ and Copeland ${ }^{1}$ from [7] to all rationals $\alpha, 0 \leq \alpha \leq$ 1: Copeland $\mathrm{Irrational}^{\alpha}$ is vulnerable to destructive microbribery. Informally put, microbribery means that the briber pays separately for each preference-table entry flip of irrational voters.

All proofs of Sections 3.4 and 4 are omitted due to space, but can be found (along with other omitted proofs) in the currently 82-page, in-preparation full version of this paper. 


\section{Conclusions}

In this paper we studied Copeland ${ }^{\alpha}$ elections with respect to their resistance and vulnerability to control and bribery. Among the election systems whose winners can be determined in polynomial time, we identified the first natural election system, Copeland (i.e., Copeland ${ }^{0.5}$ ), that provides full resistance to constructive control. Using this result, we also obtained the first (hybrid) election system that is resistant to each type of constructive and destructive control, has a polynomialtime winner problem, and is built only from natural election systems. In addition, we extended previous resistance results on bribery and fixed-parameter tractability of bounded-case control to Copeland $^{\alpha}$ for each rational $\alpha, 0<\alpha<1$. Regarding the latter, questions that remain open concern the rational-voter, candidate-control, bounded-voter cases. Another open question regards the complexity of constructive microbribery for Copeland $\mathrm{Irrational}^{0.5}$.

\section{References}

[1] J. Bartholdi, III, C. Tovey, and M. Trick. Voting schemes for which it can be difficult to tell who won the election. Social Choice and Welfare, 6(2):157-165, 1989.

[2] J. Bartholdi, III, C. Tovey, and M. Trick. How hard is it to control an election? Mathematical and Computer Modeling, 16(8/9):27-40, 1992.

[3] V. Conitzer, T. Sandholm, and J. Lang. When are elections with few candidates hard to manipulate? Journal of the ACM, 54(3):Article 14, 2007.

[4] A. Copeland. A 'reasonable' social welfare function. Mimeographed notes from a Seminar on Applications of Mathematics to the Social Sciences, University of Michigan, 1951.

[5] R. Downey and M. Fellows. Parameterized Complexity. Springer-Verlag, 1999.

[6] P. Faliszewski, E. Hemaspaandra, and L. Hemaspaandra. The complexity of bribery in elections. In Proc. AAAI'06, pages 641-646. AAAI Press, 2006.

[7] P. Faliszewski, E. Hemaspaandra, L. Hemaspaandra, and J. Rothe. Llull and Copeland voting broadly resist bribery and control. In Proc. AAAI'07, pages 724-730. AAAI Press, 2007.

[8] E. Hemaspaandra, L. Hemaspaandra, and J. Rothe. Anyone but him: The complexity of precluding an alternative. Artificial Intelligence, 171(5-6):255-285, 2007.

[9] E. Hemaspaandra, L. Hemaspaandra, and J. Rothe. Hybrid elections broaden complexity-theoretic resistance to control. In Proc. IJCAI'07, pages 1308-1314. AAAI Press, 2007.

[10] H. Lenstra, Jr. Integer programming with a fixed number of variables. Mathematics of Operations Research, 8(4):538-548, 1983.

[11] V. Merlin and D. Saari. Copeland method II: Manipulation, monotonicity, and paradoxes. Journal of Economic Theory, 72(1):148-172, 1997.

[12] A. Procaccia, J. Rosenschein, and G. Kaminka. On the robustness of preference aggregation in noisy environments. In Proc. AAMAS'07, pages 416-422. ACM Press, 2007.

[13] A. Procaccia, J. Rosenschein, and A. Zohar. Multi-winner elections: Complexity of manipulation, control, and winner-determination. In Proc. IJCAI'0\%, pages 1476-1481. AAAI Press, 2007.

[14] D. Saari and V. Merlin. The Copeland method I: Relationships and the dictionary. Economic Theory, $8(1): 51-76,1996$. 